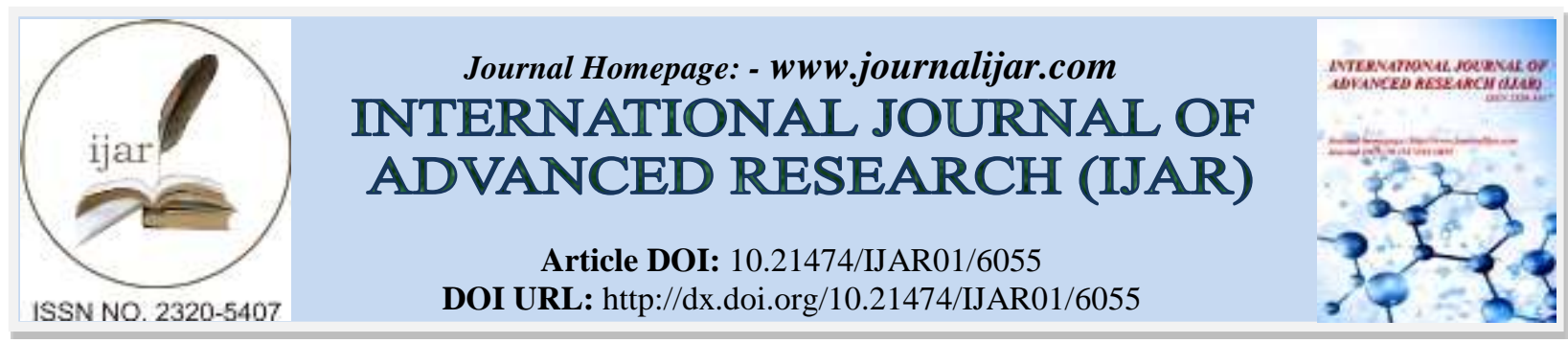

RESEARCH ARTICLE

\title{
INFLUENCE DE L'ÉCHANCRURE INTERCONDYLIENNE SUR LA RUPTURE DU LIGAMENT CROISÉ ANTÉRIEUR [ÉTUDE ANATOMORADIOLOGIQUE À PROPOS DE 60 CAS].
}

\section{Monsef El abdi ${ }^{1}$, Mohamed Reda Ouzaa ${ }^{1}$ and Abdelouahab Jaafar ${ }^{1}$.} Service de Traumatologie Orthopedie HMIMV, Rabat, Maroc.

\section{Manuscript Info}

Manuscript History

Received: 14 October 2017

Final Accepted: 16 November 2017

Published: December 2017

Key words:-

ligament croisé antérieur, échancrure intercondylienne, imagerie par résonance magnétique.

\begin{abstract}
La rupture du ligament croisé antérieur [LCA] est due à plusieurs facteurs, certains sont intrinsèques, d'autres sont extrinsèques. L'échancrure intercondylienne représente un facteur de risque étroitement lié à cette lésion du LCA. Nous rapportons une étude « castémoin » rétrospective comparant un échantillon de 30 patients consultant pour rupture unilatérale récente du LCA à un échantillon de 30 patients consultant pour des gonalgies sans rupture du LCA.

Le but de ce travail est d'étudier la relation entre l'échancrure intercondylienne et la rupture du LCA, préciser la place de l'imagerie par résonance magnétique, et d'apporter une étude supplémentaire qui intéresse un échantillon de population méditerranéenne et africaine.
\end{abstract}

Copy Right, IJAR, 2017,. All rights reserved

\section{Introduction:-}

Le genou est l'articulation la plus exposée dans la pratique sportive du fait de sa complexité anatomique, avec une stabilité assurée en majorité par le système ligamentaire et par la congruence des surfaces ostéo-cartilagineuses.

Le ligament croisé antérieur [LCA] est un élément fondamental de la stabilité sagittale et rotatoire, sa rupture est une cause d'instabilité et peut entamer une course d'évènements: lésions méniscales, arthrose, instabilité chronique......etc.

Les facteurs de risque prédisposant à la rupture du LCA incluent des facteurs intrinsèques liés à l'anatomie propre $\mathrm{du}$ genou, d'autres sont extrinsèques en rapport avec les traumatismes, surtout sportifs.

L'échancrure inter-condylienne [EIC] constitue l'environnement ostéo-cartilagineux du LCA, et représente un facteur anatomique nuisible au LCA.

\section{Matériels et Méthodes:-}

Nous rapportons une étude cas- témoin rétrospective portant sur 60 genoux répartis en 2 groupes, colligée dans le service de chirurgie traumatologique et orthopédique de l'hôpital militaire d'instruction Mohammed « V » en 12 mois [2013]. Le « groupe cas » comporte 30 ruptures unilatérales récentes [<1an] du LCA, et le « groupe témoin» avec 30 gonalgies sans rupture du LCA. Aucun de ces genoux n'avait de signe radiographique d'arthrose ni chirurgie préalable. 
Le principe de la méthode est de comparer les EIC sur 60 IRM du genou en fonction de la présence ou non d'une rupture du LCA.

Les 60 épiphyses fémorales ont été étudiées selon le même protocole [IRM du genou en extension, patient couché et reconstruites en séquences $\mathrm{T} 1$ et $\mathrm{T} 2$ avec des coupes millimétriques frontales]. Nous avons réalisé 2 mesures millimétriques:

La largeur de l'EIC passant par le centre de l'échancrure et orthogonal à l'axe du tibia sur la coupe frontale [échancrure frontale].

La largeur de l'épiphyse fémorale passant par la tangente au toit de l'échancrure et orthogonal à l'axe du tibia sur la coupe frontale [épiphyse frontale].

À partir de ces mesures, nous avons calculé le rapport frontal inspiré du NWI [Notch Width Index=index de profondeur de l'échancrure] décrit par SOURYAL [figure 1]:

$\mathrm{NWI}=\frac{\text { largeur de l'ouverture antérieure de l'EIC [échancrure frontale] }}{\text { largeur totale des } 2 \text { condyles [épiphyse frontale] }}$

L'analyse des données statistiques a été réalisée par le Logiciel SPSS 10.

Les tests statistiques ont été choisis en comparant les variables entre deux groupes indépendents. [t student, Khi 2, exact de Fisher, etc...]

Le seuil de significativité «p » a été fixé à 0,05 .

\section{Résultats:-}

Nous avons inclus 60 patients, dont 57 hommes et 3 femmes, l'âge moyen était de $35 \pm 4$ ans. La moyenne des rapports frontaux calculée à partir des mesures réalisées sur les IRM des 60 fémurs était de 0,25 [minimum=0,20 et maximum $=0,31]$. [Tableau 1].

Les moyennes des échancrures frontales et des rapports frontaux étaient plus petites dans le groupe de cas par rapport au groupe témoin. Cette différence était statistiquement significative avec un $\mathrm{p}$ respectif de $\mathrm{p}=0,03$ et $\mathrm{p}=0,02$. Par ailleurs les moyennes des épiphyses frontales étaient plus grandes dans le groupe de cas. Cette différence n'était pas significative [ $\mathrm{p}=0,09]$. [Tableau 2].

L'utilisation du test de corrélation a montré une forte corrélation entre l'échancrure frontale et le rapport frontal $[\mathrm{R}=$ $+0,763]$.

Par ailleurs, dans le groupe de cas, nous avons trouvé que les traumatismes avec contact étaient 23 [76,66\%], alors que ceux sans contact étaient $7[23,33 \%]$.

Tableau 1:- Résultats Descriptifs De La Population Étudiée.

\begin{tabular}{|l|l|}
\hline Caractéristiques & Valeurs $(\mathrm{n}=60)$ \\
\hline Age (année) & $35 \pm 4$ ans \\
\hline Sexe & 57 Hommes $(95 \%) / 3$ Femmes (5\%) \\
\hline Rapport Frontal global & $0,25 \pm 0,11$ \\
& $\min =0,20 \quad \max =0,31$ \\
\hline
\end{tabular}

Tableau 2:- Étude Analytique De La Population Étudiée.

\begin{tabular}{|c|c|c|c|}
\hline Moyennes & Groupe des Cas & Groupe des témoins & $p$ \\
\hline Echancrures frontales & $\begin{array}{l}18,40 \pm 1,79 \\
\min =14,46 \quad \max =20,80\end{array}$ & $\begin{array}{l}21,49 \pm 2,23 \\
\operatorname{Min}=16,00 \quad \max =24,80\end{array}$ & 0,03 \\
\hline Epiphyses frontales & $\begin{array}{l}74,41 \pm 3,75 \\
\min =67,70 \max =79,30\end{array}$ & $\begin{array}{l}72,94 \pm 6,10 \\
\text { Min=61,00 } \max =83,00\end{array}$ & 0,09 \\
\hline $\begin{array}{l}\text { Rapports frontaux }= \\
\text { Echancrure/Epiphyse }\end{array}$ & $\begin{array}{l}0,24 \pm 0,19 \\
\min =0,20 \max =0,28\end{array}$ & $\begin{array}{l}0,26 \pm 0,27 \\
\min =0,22 \max =0,31\end{array}$ & 0,02 \\
\hline
\end{tabular}




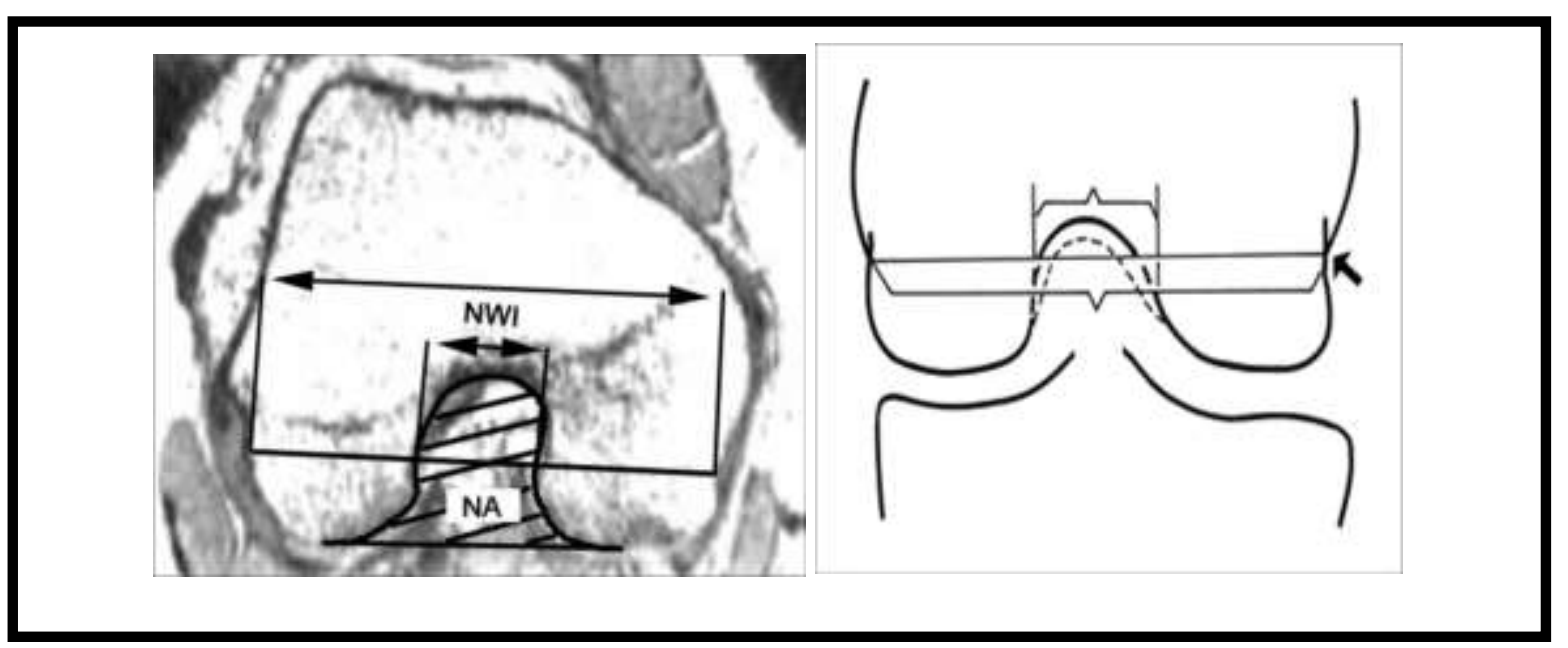

\section{Discussion:-}

Le genou est une articulation intermédiaire portante du membre inférieur avec une grande mobilité. Le LCA est un élément fondamental de la stabilité du genou, ce ligament est situé en profondeur, oblique en haut, en arrière et en dehors, se fixe sur la surface pré-spinale au niveau tibial et la face postéro-latérale du condyle fémoral interne au niveau de l'EIC [1-2]. L'IRM réalisée avec genou en extension est la technique d'imagerie de référence du LCA, elle a l'avantage d'éviter le problème de superposition inhérent à la radiologie standard et l'absence d'irradiation comparée au scanner [1-5]. L’EIC désigne la dépression séparant les surfaces condyliennes, et contient les ligaments croisés antérieur et postérieur. Ce facteur endo-articulaire doit maintenir son anatomie et ses dimensions normales pour permettre la fonction normale du LCA [3-6].

Dans la littérature les séries ont rapporté une étroitesse de l'échancrure chez les patients ayant subit une rupture du LCA, et ont évoqué une relation pathologique entre la morphologie de l'EIC et la lésion du LCA [3, 5,7-12]. Certains auteurs ont montré qu'il existe une relation géométrique entre les mesures de l'EIC-LCA, et qu'un NWI bas est un facteur prédictif de rupture du LCA [3,7-9,13-15]. D'autres ont suggéré une plastie de l'échancrure lors des ligamentoplasties du LCA [9,13-14,16-19].

Notre étude a renforcé les résultats de la majorité des études réalisées puisque Les rapports calculés de la largeur de l'échancrure inter-condylienne rapportée à la largeur de l'épiphyse fémorale dans le plan frontal sont statistiquement plus petits dans le groupe avec rupture du LCA comparés au groupe sain, ce qui prouve que les genoux avec une échancrure étroite sont les plus vulnérables à léser leurs LCA. De même, il n'ya pas de différence statistiquement significative entre les rapports calculés au sein du groupe1 en fonction du mécanisme de la rupture du LCA (traumatisme avec ou sans contact), ce qui nous amène à ne pas utiliser le mécanisme causal pour prédire de l'étroitesse de l'échancrure. Néanmoins, on n'a pas trouvé de corrélation statistiquement significative entre les mesures acquises et le sexe.

\section{Conclusion:-}

LCA serait plus vulnérable si EIC est étroite, notre étude a confirmé statistiquement la relation forte entre les valeurs de ce facteur anatomique et la rupture du LCA, ainsi notre analyse doit servir pour optimiser les résultats de la ligamentoplastie et limiter les risques de rupture itérative ou controlatérale du LCA.

\section{Conflits d'intérêts:-}

Les auteurs ne déclarent aucun conflit d'intérêts 


\section{Références:-}

1. Cha JH' ${ }^{1}$, Lee SH, Shin MJ, Choi BK, Bin SI. Relationship between mucoid hypertrophy of the anterior cruciate ligament (ACL) and morphologic change of the intercondylar notch: MRI and arthroscopy correlation. Skeletal Radiol. 2008 Sep; 37(9):821-6. doi: 10.1007/s00256-008-0527-3. Epub 2008 Jul 16.

2. Davis $\mathrm{TJ}^{1}$, Shelbourne KD, Klootwyk TE. Correlation of the intercondylar notch width of the femur to the width of the anterior and posterior cruciate ligaments. Knee Surg Sports Traumatol Arthrosc. 1999;7(4):209-14.

3. Anderson $\mathrm{AF}^{1}$, Dome DC, Gautam S, Awh MH, Rennirt GW. Correlation of Anthropometric Measurements, Strenght, Anterior cruciate ligament Size, and Intercondylar notch Characteristics to sex differences in anterior cruciate ligament tear rates. Am J Sports Med. 2001 Jan-Feb;29(1):58-66.

4. Murshed KA ${ }^{1}$, Ciçekcibaşi AE, Karabacakoğlu A, Seker M, Ziylan T. Distal femur morphometry: a gender and bilateral comparative study using magnetic resonance imaging. Surg Radiol Anat. 2005 Apr; 27(2):108-12. Epub 2004 Dec 2.

5. Buzzi $\mathrm{R}^{1}$, Zaccherotti G, Giron F, Aglietti P. The relationship between the intercondylar roof and the tibial plateau with the knee in extension: relevance for tibial tunnel. Placement in anterior cruciate ligament reconstruction. Arthroscopy. 1999 Sep;15(6):625-31.

6. Staeubli $\mathrm{HU}^{1}$, Adam O, Becker W, Burgkart R.Anterior cruciate ligament and intercondylar notch in the coronal oblique plane:anatomy complemented by magnetic resonance imaging in cruciate ligament -intact knees. Arthroscopy. 1999 May;15(4):349-59.

7. Fung $\mathrm{DT}^{1}$, Zhang LQ. Modeling of ACL impingement against the intercondylar notch. Clin Biomech (Bristol, Avon). 2003 Dec; 18(10):933-41.

8. Tillman MD ${ }^{1}$, Smith KR, Bauer JA, Cauraugh JH, Falsetti AB, Pattishall JL. Differences in three intercondylar notch geometry indices between males and females: a cadaver study. Knee. 2002 Feb; 9(1):41-6.

9. Shelbourne $\mathrm{KD}^{1}$, Facibene WA, Hunt JJ. Radiographic and intraoperative intercondylar width measurments in men and women with unilateral and bilateral anterior cruciate ligament tears. Knee Surg Sports Traumatol Arthrosc. 1997;5(4):229-33.

10. Anderson $\mathrm{AF}^{1}$, Anderson $\mathrm{CN}$, Gorman TM, Cross MB, Spindler KP. Radiographic measurements of the intercondylar notch: are they accurate? Arthroscopy. 2007 Mar;23(3):261-8, 268.e1-2.

11. Lewis $\mathrm{BA}^{1}$, Allen DA, Henrikson TD, Lehenbauer TW. Computed tomographic evaluation of the canine intercondylar notch in normal and cruciate deficient stifles. Vet Comp Orthop Traumatol. 2008;21(2):119-24.

12. Ireland $\mathrm{ML}^{1}$, Ballantyne $\mathrm{BT}$, Little $\mathrm{K}$, McClay IS. A radiographic analysis of the relationship between the size and shape of the intercondylar notch and anterior cruciate ligament injury. Knee Surg Sports Traumatol Arthrosc. 2001 Jul;9(4):200-5.

13. Lund-Hanssen $\mathrm{H}^{1}$, Gannon J, Engebretsen L, Holen KJ, Anda S, Vatten L. Intercondylar notch width and the risk for anterior cruciate ligament rupture. A case-control study in 46 female handball players. Acta Orthop Scand. 1994 Oct; 65(5):529-32.

14. Yoo JH ${ }^{1}$, Yi SR, Son BK. The prone kneeling view of the intercondylar notch for radiographic assessment of the femoral tunnel position in anterior cruciate ligament reconstruction. Arthroscopy. 2008 Apr;24(4):465-71. doi: 10.1016/j.arthro.2007.09.017. Epub 2008 Jan 7.

15. Hernigou $\mathrm{P}^{1}$, Garabedian JM. Intercondylar notch width and the risk for anterior cruciate ligament rupture in the osteoarthritic knee: evaluation by plain radiography and CT scan. Knee. 2002 Dec;9(4):313-6.

16. Dahlstedt $\mathrm{L}^{1}$, Dalén N, Dahlborn M, Nilsson T. Value of intercondylar notch platy CT studies and peroperative measurements of 127 knees. Acta Orthop Scand. 1990 Dec; 61(6):558-61.

17. León $\mathrm{HO}^{1}$, Blanco CE, Guthrie TB, Martínez OJ. Intercondylar notch stenosis in degenerative arthritis of the knee. Arthroscopy. 2005 Mar;21(3):294-302.

18. F. Ben Hamida ${ }^{1}$, S. Mourali, Z. Ben Lakhdar, M. HadjsalahF. Influence des facteurs intrinséques anatomiques dans la rupture du ligament croisé antérieur: étude anatomoradiologique comparative. Journal de traumatologie du sport septembre 2008 ; 25(3):144-147.

19. Mann TA ${ }^{1}$, Black KP, Zanotti DJ, Barr M, Teater T. The natural history of the intercondylar notch after notchplasty. Am J Sports Med. 1999 Mar-Apr; 27(2):181-8. 